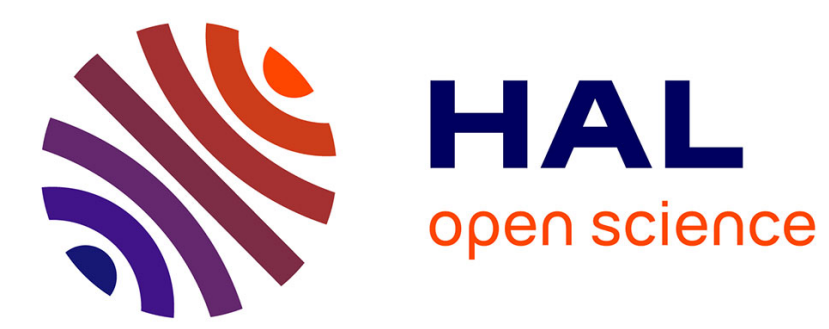

\title{
Objectiver l'intime
}

Gilles Louÿs

\section{To cite this version:}

Gilles Louÿs. Objectiver l'intime : éléments d'une poétique du moins dans chronique japonaise de Nicolas Bouvier. Poétique : revue de théorie et d'analyse littéraire, 2011, 4 (168), pp.493-502. 10.3917/poeti.168.0493 . hal-01407630

\section{HAL Id: hal-01407630 \\ https://hal.parisnanterre.fr/hal-01407630}

Submitted on 4 Dec 2017

HAL is a multi-disciplinary open access archive for the deposit and dissemination of scientific research documents, whether they are published or not. The documents may come from teaching and research institutions in France or abroad, or from public or private research centers.
L'archive ouverte pluridisciplinaire HAL, est destinée au dépôt et à la diffusion de documents scientifiques de niveau recherche, publiés ou non, émanant des établissements d'enseignement et de recherche français ou étrangers, des laboratoires publics ou privés. 


\section{Objectiver l'intime : éléments d'une poétique du moins dans Chronique japonaise de Nicolas Bouvier}

Gilles Louÿs
Article paru dans la revue Poétique, n 168 , novembre 2011, p. 493-502. ISBN 978-2-02-104027-2

"Celui qui ici n'accepte pas de commencer par faire l'apprentissage du moins est certain de perdre son temps. „ ${ }^{1}$

"(...) Le pays et l'été m'ont déjà gobé comme un ouf, ne laissant que la coquille, et je vois mal ce que je pourrais faire de plus pour lui et comment exister moins. ${ }^{2}$

A plusieurs reprises Nicolas Bouvier s'est exprimé sur la dimension ascétique que revêtait pour lui l'expérience du voyage : un exercice d'usure, de dépouillement pour parvenir à se réduire à l'essentiel et atteindre un état de disponibilité totale. Le choix de la comparaison avec les serviettes des bordels «élimées par les lessive » qu'il utilisait dans le Poisson-Scorpion montrait de façon frappante jusqu'où pouvait aller cette aptitude à se dégarnir de soi ${ }^{3}$. Idéal à rapprocher certes de celui qui pousse, depuis des millénaires, les aspirants à la sainteté sur les routes, et qui vise à rapprocher le plus possible la vie de la mort, voire à les faire quasi coïncider dans l'épreuve de l'exténuation ${ }^{4}$, afin d'accéder à l'illumination intérieure : «Un pas vers le moins est un pas vers le mieux $»^{5}$. Tout voyage pour Bouvier est cet apprentissage du dépouillement qui seul permet d'accéder à une « seconde naissance» ${ }^{6}$.

En résultent des conséquences quand vient le moment de transmettre cette expérience - et l'on sait à quel point ce travail d'écriture a pris du temps chez Bouvier ${ }^{7}$. Loin de tout pittoresque comme de toute effusion de soi, la relation du voyage se doit d'être à la hauteur de cette exigence de dépouillement: comment faire pour transmettre, de la seule façon acceptable, c'est-à-dire sobrement et efficacement, l'expérience physiologique et psychique du dépaysement à quoi se ramène le vécu du voyage ? Comment faire pour ne rien épancher du moi tout en rendant sensible ses remuements intimes ? Comment dire d'un seul tenant tout à la fois les circonstances externes et 
l'illumination intime, «le dehors et le dedans $»^{8}$ ? Par quel travail de mise en texte parvenir à une poétique du moins?

Le Chapitre XXIII de Chronique japonaise, «Le cap Erimo » constitue un lieu privilégié pour prendre la mesure de ce travail. Ce récit du voyage fait dans l'île d'Hokkaïdo au nord du Japon présente apparemment toutes les caractéristiques du récit de voyage classique. Il se donne à lire comme une sorte de carnet de route, où la progression dans l'espace, la transmission des expériences sensorielles et la relation des échanges sur place sont données à travers un découpage de cinq courtes séquences textuelles : 1/ un texte liminaire sans titre relatant le voyage nocturne en bus ; 2/ «Onze heures du matin»; 3/ «Village d'Erimo, midi»; 4/ «Entre Kushiro et? (cinq heures du matin) »; 5/ «Au lac Mashu».

Ce balisage offre au lecteur les repérages classiques du carnet de voyage et sacrifie ainsi au pacte d'authenticité requis dans ce type d'écrit. S'y ajoute le fait que le matériau du voyage, par sa pauvreté et sa banalité apparente, accroît encore cette lisibilité : un long voyage en bus la nuit, la vision à l'aube d'étendues vertes traversées de brume, un gros cheval noir qui broute, un groupement de pauvres maisons, un couple de pêcheurs rencontrés à « Onze heures du matin ${ }^{9}$, la rencontre d'une jeune fille étonnamment maquillée dans une boutique de laitier, le voyage en train omnibus depuis Kushiro en compagnie de vieilles Japonaises particulièrement décontractées... Si l'on excepte la dimension romanesque apportée par le personnage de la séduisante jeune fille aux yeux «gris violet» (« elle dressait des otaries savantes dans une boutique foraine du port de Muroran ${ }^{10}$ ), on pourrait croire à la banalité de la «chose vue », dont on sait la fonction d'accréditation d'authenticité dans la littérature de voyage. Pourtant, dans cette séquence classique, où l'on retrouve d'autres topoï incontournables de cette littérature (comme le motif de l'inconfort du voyage nocturne en bus, bien rendu par la notation des "passagers qui dorment dans des postures de suppliciés $»^{11}$ ) apparaissent, paradoxalement à des endroits où le récit pointe l'extrême dénuement du milieu naturel et humain, des indices explicites d'émotion : "Cela fait des années que, sans le savoir, j'attendais ces images qui me remuent inexplicablement $»^{12}$. Ou encore : «C'est seulement ici et aujourd'hui que j'ai compris ce que j'étais venu chercher sur cette île » ${ }^{13}$. Preuve 
que quelque chose d'extraordinaire dans cet humble décor attendait le voyageur, un extraordinaire qu'il fallait mettre à la portée du lecteur par des moyens appropriés à cette humilité. Ce sont ces moyens que l'on cherchera à identifier ici.

\section{Complexification narrative et détemporalisation de l'expérience vécue}

Une première façon de faire tient bien sûr au recours au carnet de route. Mais cette mise en récit simple et apparemment conforme au code le moins élaboré de la littérature de voyage, avec le choix du temps verbal du présent, donnant à croire à une relation au plus près de l'expérience vécue, est en réalité le produit d'une élaboration particulièrement complexe, visible au niveau de l'énonciation et de la technique narrative.

\subsection{La dépersonnalisation de l'expérience}

La distribution des pronoms dans le texte (on passe du «nous » initial au «je » puis à «on» repris par un «vous» qui n'est pas un «vous »d'interlocution mais la forme remplaçant «on » en fonction de complément du verbe, ce que les grammairiens appellent le «vous » indéfini ${ }^{14}$ ) attire l'attention sur des relais successifs de focalisation : si le «nous » employé au début atteste que le voyageur-narrateur s'énonce comme partie prenante des passagers du bus («nous sommes une douzaine là-dedans à lutter contre le sommeil $»^{15}$ ), puis qu'il singularise sa perspective à travers le pronom «je» («Je me demande comment le chauffeur de l'autobus peut traverser chaque jour ce

palais de glaces ... ${ }^{16}$ ), à plusieurs reprises, le glissement $\mathrm{du}$ «je » au pronom «on », lui-même repris par le «vous » indéfini, signale le passage à un autre point de vue : celui de toute personne qui pourrait faire, à la place du narrateur, et dans les mêmes conditions, ce type de voyage en bus, et donc vivre les mêmes mésaventures, le même inconfort, les mêmes accidents possibles. Il y a donc, dans cette oscillation de points de vue, une double articulation du voyage : l'expérience de voyage personnellement vécue et racontée par Bouvier est transmise au lecteur de telle sorte que celui-ci puisse la percevoir comme pouvant être anonymement celle de n'importe qui d'autre. Procédé grammaticalement très simple et narrativement très efficace, puisqu'il permet à n'importe quel lecteur de faire la route non pas simplement avec le voyageur, mais bien, d'une certain manière, à sa 
place $^{17}$. Cette façon de raconter une expérience vécue à la première personne tout en ayant recours à des outils grammaticaux permettant de la dépersonnaliser satisfait la double exigence de transmission et de neutralisation de l'intime, conformément au mélange de spontanéité et de retenue caractéristique du style de Bouvier. Ce procédé prend tout son sens lorsque Bouvier généralise une impression, qui ne peut être pourtant que très intime : «On est là comme dans le ventre de sa mère, plus secoué seulement ${ }^{18}$. La sensation de régression à l'état utérin créée par le fait d'être brinqueballé à l'intérieur du cocon confortable du bus n'est pas énoncée personnellement mais anonymement : façon humoristique de transmettre une expérience personnelle qui est aussi un lieu commun, quand on sait que le motif de la renaissance est un des topoï les plus récurrents de la littérature de voyage.

\subsection{La détemporalisation de l'expérience}

Le même travail de complexification peut être constaté dans la technique narrative : on croit retrouver dans le récit du voyage en bus vers le Cap Erimo le simple déroulement chronologique d'un déplacement nocturne daté et situé (récit singulatif), mais en réalité c’est un récit complexe, qui repose sur des temporalités différentes, et qu'on a du mal à distinguer puisque le temps verbal utilisé est le présent. Bouvier a en effet recours, à plusieurs reprises, au récit itératif, qui relie 1'expérience du voyage en bus au Cap Erimo à celle d'autres voyages antérieurs à travers l'archipel effectués dans les mêmes conditions («J'en ai pris si souvent. Tout cela m'est si familier (...)» ${ }^{19}$ ), de telle sorte qu'à ce voyage singulier se superpose le souvenir d'autres voyages analogues, avec le rappel des mêmes sensations, des mêmes détails. Il existe une sorte de mémoire corporelle du voyage en bus, tellement forte, que nommer les sensations du voyage au Cap Erimo suffit à convoquer les sensations semblables vécues ailleurs et en d'autres temps sur l'archipel, que Bouvier fait surgir dans son récit par une simple énumération : «le ceinturon trop serré de la receveuse qui lui fait bomber le ventre, ses coups de sifflets pour aider à la manœuvre, (...), la rose de plastique dans un vase au-dessus du conducteur, le micro qui merdoie un peu et qu'on tapote sans succès pour le faire taire, les passagers qui dorment dans des postures de suppliciés. Ces choses je les 
connais si bien que je ne les vois même plus (...). On est là comme dans le ventre de sa mère, plus secoué seulement... $»^{20}$

Par cette alternance de récit singulatif et de récit itératif, Bouvier détemporalise le souvenir de son voyage vers le Cap Erimo en en faisant le condensé d'une même et unique expérience : plusieurs voyages co-présents se superposent ou s'enchevêtrent, tous semblables par ce qui revient à l'identique dans ce type de transport: non seulement l'inconfort, la somnolence, l'ennui, la promiscuité, mais également les risques d'accidents : «(Le bus), cela va partout. Quelquefois même dans le ravin, dans la rivière, au bas de la falaise rongée par les vagues, et (...) alors adieu la vie, ou pis encore, adieu les femmes ou, si c'est la tête qui écope, adieu les problèmes d'hectolitres et les citations qu'on place au bon moment. » ${ }^{21}$

Il se produit alors dans le récit de Bouvier quelque chose d'étonnant : pour montrer que même cette épreuve peut être considérée comme un bienfait du voyage, il use d'une forme concessive («Mais quand même ce malheur vous arriverait, quand même vous ne distingueriez plus le Yin du Yang ni le Fils du Saint-Esprit... » ${ }^{22}$ ) pour introduire le récit par anticipation de ce qui arriverait à un voyageur victime d'un accident de bus : le personnel de hôpital qui « se mettra en quatre », les brancardiers qui «vous feront des papiers pliés en forme d'hirondelle ou de baleine pour vous arracher un sourire », la visite du journaliste local « un ex-officier de la marine impériale peut-être, qui ne sait pas bien manier son flash», puis «dans la gazette locale le soustitre qui vous concerne ( "Destin tragique d'un visiteur étranger») », et enfin «sur votre lit ... les lettres d'écolières désolées, sincères, brûlantes » que le professeur de français «le plus proche viendra sur sa moto vous traduire (...), des lettres telles que le jeune Werther n'en écrivit jamais et telles que vous nous recevrez jamais plus... ${ }^{23}$.

Par cet étonnant récit au futur, Bouvier fait vivre à son lecteur ce qui ne manquerait pas de lui arriver s'il se retrouvait dans la situation décrite par son texte, avec force détails - toutes ces marques extraordinaires de sollicitude à l'égard de l'infortuné visiteur étranger - qui semblent s'appuyer sur une expérience vécue: en particulier, l'anecdote des lettres d'écolière «inoubliables ${ }^{24}$ traduites par le professeur de français venu « sur sa moto » semble transmettre un 
souvenir vécu, une expérience authentique ... Bouvier s'est-il lui-même retrouvé dans cette situation du visiteur étranger sur un lit d'hôpital croyant dire adieu «aux problèmes d'hectolitres »? Dans ce récit au futur il faut en effet comprendre que l'anticipation de ce qui se passerait en cas d'accident de bus - toutes les manifestations de ce touchant intérêt des Japonais pour l'hôte étranger - postule une connaissance de ce qui se passe fréquemment au Japon dans ce cas, et donc une expérience vécue de voyageur visiblement attestée par ce texte. Est-elle celle de Bouvier lui-même (éventuellement affabulatrice) ? Celle d'un autre voyageur étranger dont il aurait lu l'histoire (par exemple dans une «gazette locale», précisément) ? Qu'est-ce qui permet au narrateur de ce récit (masqué derrière le «on » et le «vous») de promettre à toute personne faisant le même type de voyage en bus au Japon qu'elle pourrait vivre ce type d'expérience inoubliable ? Toujours est-il que Bouvier invente là un peu usité récit itératif au futur ${ }^{25}$, lequel, couplé avec l'anonymat qui affecte le point de vue utilisé dans la narration, a pour effet d'opérer la dépersonnalisation et la détemporalisation de l'expérience vécue.

On a ici une façon très originale d'exprimer une expérience singulière en l'attribuant à n'importe qui, ce qui permet précisément de résoudre l'équation première de Bouvier, à savoir transmettre quelque chose de très personnel tout en mettant à distance, précisément, ce qu'il y a de personnel et d'intime. A ceci s'ajoute le procédé de mise à distance par l'humour, constamment visible dans l'évocation des dégâts infligés par les accidents de bus, mais aussi à travers la comparaison interculturelle établissant la supériorité de l'art épistolaire des écolières japonaises sur celui de l'immortel Goethe.

\section{Objectiver l'émotion}

\subsection{L'expression de l'affinité avec le paysage}

Stendhal a décrit dans Vie de Henri Brulard ce bonheur extrême qui saisit à la vue du paysage romain, un bonheur qui coupe le souffle et qui submerge. Plus encore que de la perception des formes harmonieuses et de la vivacité des couleurs, l'euphorie stendhalienne se nourrit de ce que rappelle à l'œil et l'esprit la contemplation des monuments des siècles passés : l'Histoire en 
quelque sorte rendue visible, confondue avec la matière, la lumière, et réunie à l'instant. De nombreux signes d'expressivité - le «style coupé » des phrases exclamatives - témoignent dans le récit de Stendhal de cette émotion, que son texte ne cherche nullement à dissimuler mais au contraire à communiquer de la manière la plus explicite, puisque, de cette contemplation muette de l'Histoire et de la Beauté romaines naît par contrecoup le désir de répondre à la fameuse question "Qu'ai-je été?».

Il se produit dans le récit de Bouvier un phénomène à la fois comparable et complètement inverse lorsqu'il décrit l'arrivée à l'aube au Cap Erimo et la découverte du paysage environnant. A la différence du paysage urbain saturé de civilisation et d'humanité que décrit Stendhal, avec une telle netteté qu'on a l'impression que les infimes détails vus de loin le sont à travers une longue vue, ce qu'entrevoit Bouvier au Cap Erimo est un espace nu, sommaire, une vague marqueterie de vert et de bleu quasi constamment recouverte par d'épaisses nappes de brume «d'une blancheur hypnotique $»^{26}$. Il n'y a donc rien à voir à proprement parler dans cette étendue strictement naturelle et sauvage, si ce n'est les éléments eux-mêmes (l'air, l'eau, la terre), puis, lorsque le soleil se lève, cinq maisons matérialisées par des tâches de couleur flottant dans la brume, d'autres tâches rouges ( «inq bateaux décorés d'énigmatiques motifs rouges qui ressemblent à des runes ${ }^{27}$ ) et un unique cheval noir qui broute «avec ivresse » au sommet d'une colline. C'est tout, mais cela suffit à Bouvier: tout son texte excelle à dire les fugitives apparitions de couleur et de forme dans un paysage que le brouillard persistant vise à faire disparaître. Cette atmosphère de disparition et de fin des choses est rendue notamment par le recours constant à la négation, syntaxique et lexicale, qui imprègne son texte parfois jusqu'à la saturation : «Il n'y a personne dans ce paysage fait exclusivement d'herbe, de lumière, de remous, pauvre, obstiné, répétant inlassablement la même chose comme dans un rêve » (...). ${ }^{28}$

Le brouillard lui-même est au premier plan dans ce texte qui en fait la caractéristique essentielle et remarquable d'un paysage en lui-même «pauvre ». Le brouillard est ce qui donne à ce paysage où il n'y a quasiment rien à voir son allure dramatique : il est à la fois ce qui fait disparaître et il est l'image même de la disparition et c'est en quoi il est profondément émouvant, mais, à la 
différence de l'effusion de Stendhal, il s'agit ici d'une émotion contenue, tout juste suggérée, transmise très sobrement à l'intérieur de la description elle-même, par des signes discrets qui en font une qualité inhérente au paysage lui-même et ainsi l'objectivent : par le recours à l'hypallage («des chiffons noirs ... claquent anxieusement»), à l'allusion mythologique («ce brouillard .. creusé de galeries de vent où les corbeaux s'engagent en nombre impair »), à l'intrusion "une épave, un courant, une sirène ou je ne sais quelle bête ${ }^{29}$, ou d'autres procédés à caractère indirect et banal mais singulièrement efficaces pour suggérer l'émotion sans la dire (comme par exemple le rapprochement du paysage avec la «boule de cristal magique» ou le «palais de glaces» du folklore, toutes choses évocatrices du monde de l'enfance).

Contrairement à Stendhal, dont le bonheur se nourrit de la richesse d'apparition du visible, la raison pour laquelle ce paysage émeut Bouvier tient au fait qu'il est vide, presque invisible, que parfois des touches vertes ou violettes y apparaissent comme par magie : entrer dans ce paysage, c'est quitter ce monde pour entrer dans une toile de Jérôme Bosch - mais un Jérôme Bosch qui se serait «surpassé » en débarrassant «son tableau de la rocaille, des diableries, de tout l'inutile » ${ }^{30}$ et en ne gardant que la matière même du rêve : le vide, l'invisible, l'entr'aperçu, la disparition...

Pour tout autre que Bouvier il y aurait quelque chose d'angoissant et de funèbre, dans ce paysage dont il écrit contradictoirement qu'on ne peut le traverser «sans tomber dans une sorte d'ébriété ou une mélancolie incurable ${ }^{31}$, comme si la même frugalité, le même dénuement pouvait tout aussi bien conduire à l'euphorie qu'à la dépression. Mais on notera que même lorsque son texte bascule dans la confidence ( Cela fait des années que, sans le savoir, j’attendais ces images qui me remuent inexplicablement $»^{32}$ ) son énoncé persiste à tenir l'émotion à distance grâce à un emploi figuré. Et lorsqu'il développe cette confidence, il ne peut s'empêcher d'avoir recours à une forme d'expression pareillement indirecte en suggérant seulement par les ressources de la métonymie l'intensité de son émotion : "J'ai quitté le bus et suis resté sur le bord de la route à peu près ivre, la tête bourdonnante, et de la peine à déglutir comme quelqu'un pour qui on en fait trop. J'ai poursuivi ma route à pied. $»^{33}$ Rien de tel que le langage du corps pour tout à la fois suggérer 
l'intensité d'une émotion qui vous déborde et la tenir à distance dans son expression : objectiver l'intime, tout est là.

Comme Stendhal défaillant de bonheur appuyé contre son mur romain, Bouvier a exprimé là de façon saisissante un des moments de révélation extraordinaire vécus durant ses séjours au Japon, mais il est très révélateur qu'à la différence du langage exclamatif de Vie de Henri Brulard il ait eu recours à cette poétique du moins.

\subsection{L'expression de l'affinité avec le milieu}

Il est frappant de voir à quel point la même poétique s'applique quand Bouvier s'attache à transmettre ce qu'il a ressenti au contact des milieux populaires qu'il a côtoyés dans cet extrême nord du Japon. Le récit de sa rencontre au Cap Erimo avec un couple de pêcheurs ${ }^{34}$ est exemplaire de cette discipline dans l'expression. En 27 lignes, où pas un mot n'est de trop, par le choix d'un juste réglage entre distance, empathie, sens de l'observation et connaissance des codes, Bouvier parvient à condenser toute une approche anthropologique des êtres et à témoigner en même temps, discrètement, d'un profond respect humain, d'autant plus fort qu'il n'est jamais dit explicitement.

Le lien qu'il établit entre les pêcheurs, qui vont «par couples, et jamais bien éloignés l'un de l'autre », et «les albatros ou les pluviers », lui permet d'objectiver sobrement (comme pourrait le faire un naturaliste auquel il emprunte d'ailleurs les codes) la capacité touchante à survivre dans un environnement extrême, grâce à une adaptation quasi mimétique au milieu naturel et à une solidarité sans faille.

De même, sa façon d'utiliser conjointement les ressources de son palais et celles de son intelligence pour analyser les propriétés gustatives du kombu, cette algue qui est la ressource essentielle des pêcheurs du Cap Erimo, est très révélatrice de cette capacité d'être en affinité vibratoire avec le milieu : "J'ai poursuivi ma route en mâchonnant cette espèce de cuir qui contient tous les goûts de la mer: sel, iode, la trace d'un banc d'anchois ou le sillage huileux d'un cargo. En le retournant sur la langue on a même l'impression d'y sentir la pulsation des marées et le poids de la lune. Cela m'a tenu lieu de déjeuner. » 
On retrouve certes là le goût de l'observation didactique, si caractéristique d'une certaine littérature du voyage, mais d'une façon qui n'a rien à voir avec le souci de communiquer un code gastronomique local (un des topoï de cette littérature) : la notation ici est faite de telle sorte que les mots qui désignent les saveurs vous transportent par métonymie au plus profond de la mer. On ne saurait montrer de façon plus économique la recherche du lien naturel entre l'homme et son milieu. Le commentaire terminal («Cela m'a tenu lieu de déjeuner») d'ailleurs l'atteste : manger est un acte de connaissance.

Plus loin, dans le train omnibus entre Kushiro et une destination inconnue, lorsqu'il évoque sa rencontre avec les si pittoresques marchandes de poisson dont il observe le manège dans «le wagon frugal et rapé » avec une très grande attention, mais aussi avec une sympathie non dissimulée, Nicolas Bouvier ne peut s'empêcher de corriger l'excès de sentiment qu'on pourrait lire dans ce qu'il écrit à leur sujet («c'est la compagnie la plus chaude et la plus libre que j'aie rencontré au Japon.») par ce commentaire: "Je ne dis pas cela par une sentimentalité à la Richepin, mais parce que je vois bien avec quelle aisance ces gens s'entraident, s'accordent, trouvent partout matière à rire ou à bavarder. Quand je les photographie, ils s'amusent-sans gêne ni niaiserie - parce que j'opère de tout près et qu'ils sont habitués à se retrouver minuscules sur ces photos d'albums où, par sentiment de l'insignifiance du personnage, on inclut le plus de paysage possible. » 35

Peut-être est-ce dans cette confidence, malgré tout si typiquement pleine de retenue, qu'on pourrait voir la clé du très grand attachement de Bouvier à ce peuple japonais, auprès duquel il a certainement beaucoup appris, tout en se sentant en connivence avec lui : on ne peut en tout cas s'empêcher d'établir un lien entre ce sens de la réserve et de l'insignifiance du moi, si proche de sa propre culture familiale huguenote (dont il lui est arrivé de parler, ailleurs ${ }^{36}$ ), et sa fuite loin d'un monde «où l'aspect émotif de la vie était rigoureusement géré » 37. 


\section{NOTES}

${ }^{1}$ Nicolas Bouvier, Chronique japonaise, in Euvres, édition Quarto Gallimard, 2004, p. 522. Toutes les citations de Chronique Japonaise $(C J)$ dans le présent article renvoient à cette édition.

${ }^{2}$ Ibid., p. 628.

${ }^{3}$ «On ne voyage pas pour se garnir d'exotisme et d'anecdotes comme un sapin de Noël, mais pour que la route vous plume, vous rince, vous essore vous rende comme ces serviettes élimées par les lessives qu'on vous tend avec un éclat de savon dans les bordels. » (Le Poisson-Scorpion, in Euvres, Quarto Gallimard, 2004, p. 748)

${ }^{4}$ Gérard Cogez mentionne le rôle formateur de la fatigue chez Nicolas Bouvier : "Il l'a souvent vécue comme une compagne fidèle et un véritable principe d'imprégnation, d'harmonisation avec les lieux où il a séjourné. La mise entre parenthèses de l'ego qu'elle provoque constitue une irremplaçable source de découvertes. »(Gérard Cogez, Les écrivains voyageurs au XXe siècle, éd. du Seuil, 2004, p. 197)

${ }^{5}$ Le Poisson-Scorpion, p. 748.

${ }^{6}$ Nicolas Bouvier, L'usage du monde (1963), éd. Payot \& Rivages, 2001, p. 163.

${ }^{7}$ Il a fallu 11 ans à Nicolas Bouvier pour produire le premier récit de son séjour au Japon (sous le titre Japon, en 1967 texte qui s'est enrichi à deux reprises, lors de sa réédition en 1975 sous le titre Chronique japonaise, puis en 1989, dans une version accueillant les notes de son $3^{\mathrm{e}}$ séjour de l'année 1970). Près de 10 ans séparent son grand voyage de l'année 1953-54 en Europe centrale et en Asie de la publication de l'Usage du monde en 1963. Quant à la mise en texte de son séjour apocalyptique à Ceylan, en mars-octobre 1955, il lui aura fallu pas moins de 26 ans pour la donner à lire, puisque le Poisson-Scorpion paraît en 1981.

${ }^{8}$ Le dehors et le dedans, titre d'un recueil de poèmes de Nicolas Bouvier, La Découverte, 1991.

${ }^{9}$ CJ, p. 648.

${ }^{10}$ Ibid., p. 649.

11 Ibid., p. 647.

12 Ibid., p. 648.

${ }^{13}$ Ibid., p. 649.

${ }^{14}$ Voir par exemple la définition qu'en donne Maurice Grevisse dans Le bon usage, § 725e (édition consultée : Le bon usage, $12^{\mathrm{e}}$ édition refondue par André Goosse, éd. Duculot, 1991 (1986), p. 1141.

${ }^{15}$ CJ, p. 646.

${ }^{16}$ Ibid.

${ }^{17} \mathrm{Ce}$ «vous » indéfini, reprenant «on » en position de complément, revient 16 fois dans le récit du voyage en bus pp.648-648 (soit autant que le pronom «je » qui compte 15 occurrences). Nicolas Bouvier a très souvent recours, dans Chronique japonaise comme dans ses autres textes, à cette utilisation des pronoms «on » et « vous » se substituant à la première personne. Voir par exemple dans le chapitre XV de Chronique japonaise, "Autour d'Araki-Cho », comment, par le même procédé, il associe le lecteur à ses pérégrinations dans les quartiers de Tokyo à la recherche d'un logement.

${ }^{18}$ CJ, p. 647.

${ }^{19}$ Ibid.

${ }^{20}$ Ibid.

${ }^{21}$ Ibid.

${ }^{22}$ Ibid.

${ }^{23}$ Ibid.

${ }^{24}$ Et dont Bouvier cite un échantillon : «Je suis seule dans la ferme, je pense à votre mère. Les cerisiers ont déjà fleuri ; les pruniers pas encore. Rattachez-vous aux ancêtres, aux saisons, et vous reprendrez courage. » (CJ, p. 647) la citation ayant, comme on sait, la fonction d'accréditer l'authenticité de ce dont elle parle.

${ }^{25}$ Gérard Genette remarquait que les « relations de fréquence » dans le récit constituent « un des aspects essentiels de la temporalité narrative ». (Gérard Genette, Figures III, éd. du Seuil, 1972, p. 145.)

${ }^{26}$ CJ, p. 646.

${ }^{27}$ Ibid., p. 648.

${ }^{28}$ Ibid., p. 646.

${ }^{29}$ Ibid.

${ }^{30}$ Ibid., p. 648.

${ }^{31}$ Ibid., p. 646.

${ }^{32}$ Ibid., p. 648.

${ }^{33}$ Ibid.

${ }^{34}$ CJ, pp. 648-49.

35 "Entre Kushiro et ? (cinq heures du matin) », CJ, p 650.

${ }^{36}$ Notamment dans Thesaurus Pauperum ou la guerre à huit ans (in Euvres, op.cit., pp.1233-1247) et Routes et déroutes (ibid., pp. 1249-1303).

${ }^{37}$ Jacques Meunier, « Nicolas Bouvier : l'œil du voyageur », in Le monocle de Joseph Conrad (1987), éd. Payot \& Rivages, 1993, p.244. 